\title{
Specification of real-time imaging systems using the UML
}

\author{
Colin J. Neill, Phillip A. Laplante* \\ Pennsylvania State University, Great Valley Graduate Center, 30 East Swedesford Road, Malvern, PA 19355-1443, USA
}

Received 18 June 2002; received in revised form 13 February 2003; accepted 21 February 2003

\begin{abstract}
Real-time imaging systems are expected to perform at a rate set by the operating environment. This places additional temporal constraints on the implementation, and has led to advances in parallel processing and optimization. The constraints are also applied, however, to the analysis and design models of the system, an aspect that has received far less consideration, particularly outside of academia. It is well understood that the earlier an error is discovered the cheaper the fix and this is taken to the extent that, if an error can be found during design or even analysis, the repair can be several orders of magnitude cheaper. It is therefore an economic imperative that the analysis and design models are sufficiently expressive such that the flaws in the model can be discovered before they are implemented, and this applies equally to real-time constraints.

The contribution of this paper is to make the case for the use of the Unified Modeling Language, extended with a rigorous formal method, the Q-model, for the specification and design of real-time imaging systems. To illustrate its utility the proposed approach is examined in comparison with a more traditional approach, Structured Analysis and Design, by way of a case study.

(C) 2003 Elsevier Science Ltd. All rights reserved.
\end{abstract}

Keywords: Real-time; Imaging systems; Object-oriented; Formal Method; UML

\section{Introduction}

In the last decade there has been a significant increase in the interest in real-time image processing. Indeed, since 1995 there has been an almost 100\% increase in scholarly articles devoted to the subject over that of the previous 25 years [1]. Many factors may contribute to this figure, but without question the domain of real-time imaging systems has grown and continues to grow rapidly. It is unfortunate then to find that very little of this attention has been focused on appropriate techniques or methodologies for the specification, analysis and design of such systems.

Traditionally real-time imaging systems have been specified and designed using a mix of structured modeling techniques and operational descriptions with scant regard for consistent abstractions, analyzable models or maintenance needs. This is not because structured approaches cannot cope with these issues, but rather that the user community has not adopted them; preferring pseudo-code, flowcharts or block

\footnotetext{
*Corresponding author.

E-mail address: plaplante@gv.psu.edu (P.A. Laplante).
}

diagrams [2]. It may be that this is, in part, due to a lack of understanding and/or appreciation for the software engineering practices that have been developed over the decades since the waterfall model of software development was introduced. That is to say, most experienced practitioners of imaging systems are not, in general, software engineers, but, rather, imaging engineers who construct their systems, or parts thereof, in software.

The lack of a disciplined software engineering approach is exacerbated when the special characteristics of these systems are considered. Real-time imaging systems differ from non-real-time in that the logical correctness of the system requires both correct and timely outputs [3]. Timeliness is therefore a systemic constraint-it applies across the entire system rather than to a single component or subsystem. To represent this constraint in any specification models is therefore non-trivial, yet is only one of many real-time constraints that must be included in the specification. The net effects of these constraints for image processing applications are three fundamental design trade-offs [4]:

- performance versus image resolution;

- performance versus storage; and 
- input/output bandwidth and number of tasks versus their synchronization.

It would be desirable to make these trade-off decisions some time before implementation, however. Therefore, the temporal characteristics must be present in the specification models and in a form amenable to analysis.

Looking beyond the temporal aspects, imaging systems must exhibit a high degree of the same desirable qualities as most other software-intensive systems: robustness, flexibility, generality and portability [5] and these are difficult, if not impossible, to achieve without a systematic approach to development. Interestingly, it is these very characteristics that objectoriented approaches are famed for providing [6], especially when applying design patterns such as those used later in the case study.

\section{The real-time problem}

As stated above, to support the analysis necessary to demonstrate the meeting of temporal constraints, a model of the system must include representation of temporal properties and a mechanism to demonstrate conformance and correctness. Specifically, we are interested in stipulating three aspects [7]:

- synchronization relationships between tasks;

- time-selectivity of inter-task communication (the "age" of communicated data);

- task activation and execution times.

Given these requirements very few specification techniques pass muster. In fact, the vast majority of popular software specification methods do not include time at all except perhaps as a label or annotation. To highlight this fact, it is useful to consider two methods for system specification and design, namely, Structured Analysis and Structured Design (SASD) and ObjectOriented Analysis and Design (OOAD) given their respective dominance in the field of software engineering over the last 30 years.

To illustrate the discussion an example is presented of the specification of a stereotypical real-time imaging system. This example is adapted from [8] and is for an industrial automated visual inspection (AVI) system. AVI is an interesting case since it represents a simple intuitive example of an embedded system where the temporal performance is dictated by the operating environment rather than by the computer system itself. A typical set-up is shown below in Fig. 1.

Images of the products are captured as they move along the conveyor. The images are pre-processed and then classified, using an appropriate feature-matching algorithm, as "pass" or "fail" by the system and

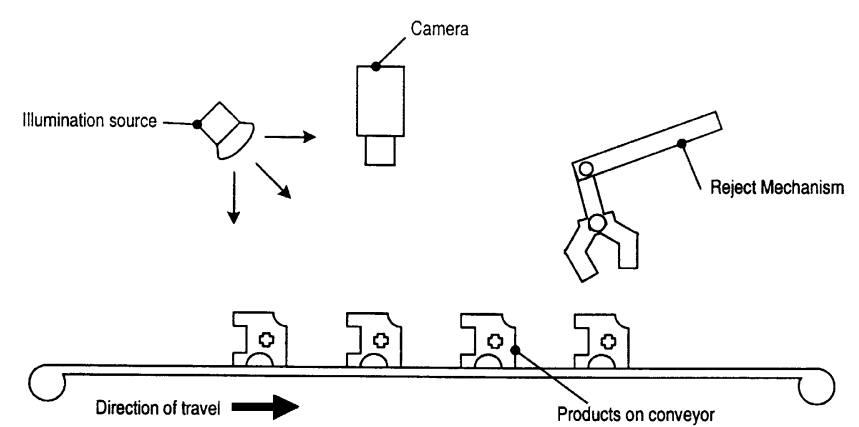

Fig. 1. AVI System.

defective products are removed from the conveyor by the reject mechanism. It is clear here that the timing constraints are imposed by the manufacturing system. The products must be classified in time for the reject mechanism to remove the product, and the system must meet the rate of arriving products. This is clearly not a safety critical system given the lack of any catastrophe if the constraints are not met, but a failure to detect faulty items (at all or in time) could certainly have serious consequences (in [8] the failure to detect a faulty lampcap could result in an electrocution if that item was used).

\section{Structured analysis and design}

Methods for SASD have evolved over almost 30 years and appear in many forms (see [9-13]) but the de facto standard is Yourdon's Modern Structured analysis [14].

Yourdon's Modern Structured Analysis uses three viewpoints to describe a system: an Environmental Model, a Behavioral Model, and an Implementation Model. The elements of each model are shown in Fig. 2.

The Environmental Model embodies the analysis aspect of SASD and consists of a context diagram and an event list. The purpose of the Environmental Model is to model the system at a high level of abstraction.

The Behavioral Model embodies the design aspect of SASD as a series of Data Flow Diagrams (DFDs), Entity Relationship Diagrams (ERDs), Process Specifications (P-SPECs), State Transition Diagrams (STDs) and a Data Dictionary. Using various combinations of these tools the designer models the processes, functions and flows of the system in detail.

Finally, in the Implementation Model the developer uses a selection of Structure Charts, natural language and pseudo-code to describe the system to a level that can be readily translated to code.

\subsection{Structured analysis}

Structured Analysis (SA) is a way to try to overcome the problems of classical analysis using graphical tools and a top-down, functional decomposition method to 


\begin{tabular}{l|c} 
Context Diagram & \multicolumn{1}{c}{$\begin{array}{c}\text { Environmental } \\
\text { Event List }\end{array}$} \\
Natural Language & $\begin{array}{c}\text { Model } \\
\text { DFD/CFD }\end{array}$ \\
ER Diagram & Behavioral \\
Data Dictionary & Model \\
P-SPEC. C-SPEC & \\
STD/FSM & \\
Natural Language & \\
Structure Charts & \\
P-SPEC & Implementation \\
Temporal Logic & \\
Natural Language &
\end{tabular}

Fig. 2. Elements of Structured Analysis and Design.

define system requirements. SA is used to model a system's context (the source and sink of inputs and outputs, respectively), processes (what functions the system performs, how the functions interact, how inputs are transformed to outputs) and content (the data the system needs to perform its functions).

The target document for SA is called the Structured Specification. It consists of a system Context Diagram, an integrated set of DFDs showing the decomposition and interconnectivity of components, and an event list to represent the set of events that drive the system.

To illustrate the SA technique, consider the Visual Inspection System. Fig. 3 depicts the Context Diagram. Here, the Visual Inspection System is shown with the other constituent system parts - camera, product detector, production conveyor controller system and reject mechanism. Solid arcs indicate the flow of data between system components. In the example the only data flow involves the transmission of the captured image to the Visual Inspection System. The dashed lines represent the flow of control information. This facility is one of the extensions needed for dealing with real-time systems, which is discussed later.

In the example the event list consists of the new-product_event, which indicates the detection of the next image on the line, accept, which indicates that the product has passed inspection and causes a signal to be sent to the conveyor controller, and reject, which causes a signal to be sent that directs the conveyor to move the product into a rejected product bin. The rejection mechanism automatically causes the next product item to be moved along by the conveyor controller.

\subsection{Structured design}

Structured Design (SD) is a systematic approach concerned with the specification of the software

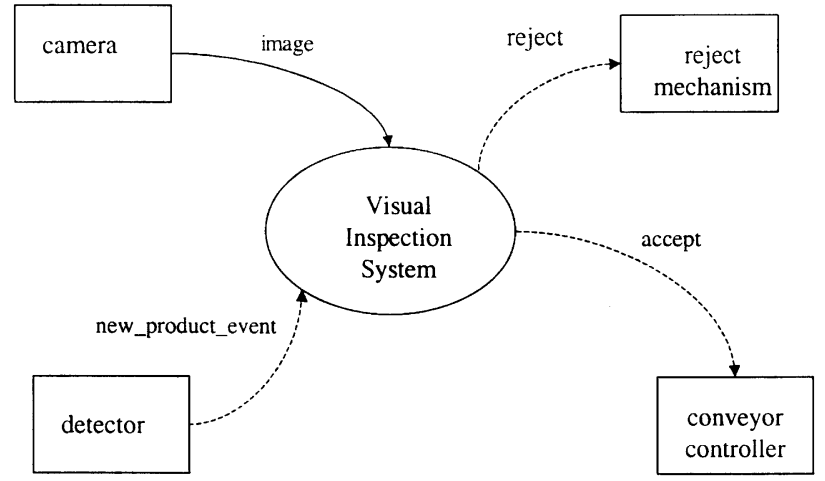

Fig. 3. Context Diagram for Visual Inspection System.

architecture and involves a number of techniques, strategies and tools. SA is related to SD in the same way that a requirements representation is related to the software architecture, that is, the former is functional and flat and the latter is modular and hierarchical.

The transition mechanisms from SA to SD are manual and involve significant analysis and trade-offs of alternative approaches. Normally, SD proceeds from SA in the following manner. Once the Context Diagram is drawn, a set of DFDs is developed. The first DFD, the level 0 diagram, shows the highest level of system abstraction. Decomposing processes to lower and lower levels until they are ready for detailed design renders new DFDs with successive levels of increasing detail.

In a typical DFD boxes represent terminators that are labeled with a noun phrase that describes the system, agent or device from which data enters or to which data exits. Each process, depicted by a circle, is labeled as a verb phrase describing the operation to be performed. Solid arcs are used to connect terminators to processes and between processes to indicate the flow of data through the system. Each arc is labeled with a noun phrase that describes the data. Dashed arcs are control flows and will be discussed later. Parallel lines indicate data stores, which are labeled by a noun phrase naming the file, database, or repository where the system stores data.

Processes at the lowest level are described by Process Specifications (P-SPECs), and are expressed in structured natural language, pseudo-code, decision tables, or decision trees and are used to describe the logic and policy of the program.

Returning to the Visual Inspection System example, Fig. 4 shows the level 0 DFD.

Here the details of the system are given at a high level. First, the system reacts to the arrival of a new product by confirming that the image data is available. Next, the system captures the image by buffering the raw data from the capture device to a file. Preprocessing of the raw data is performed to produce an image frame to be used for classification and generation of the appropriate control signals to the conveyor system. 


\section{Level 0}

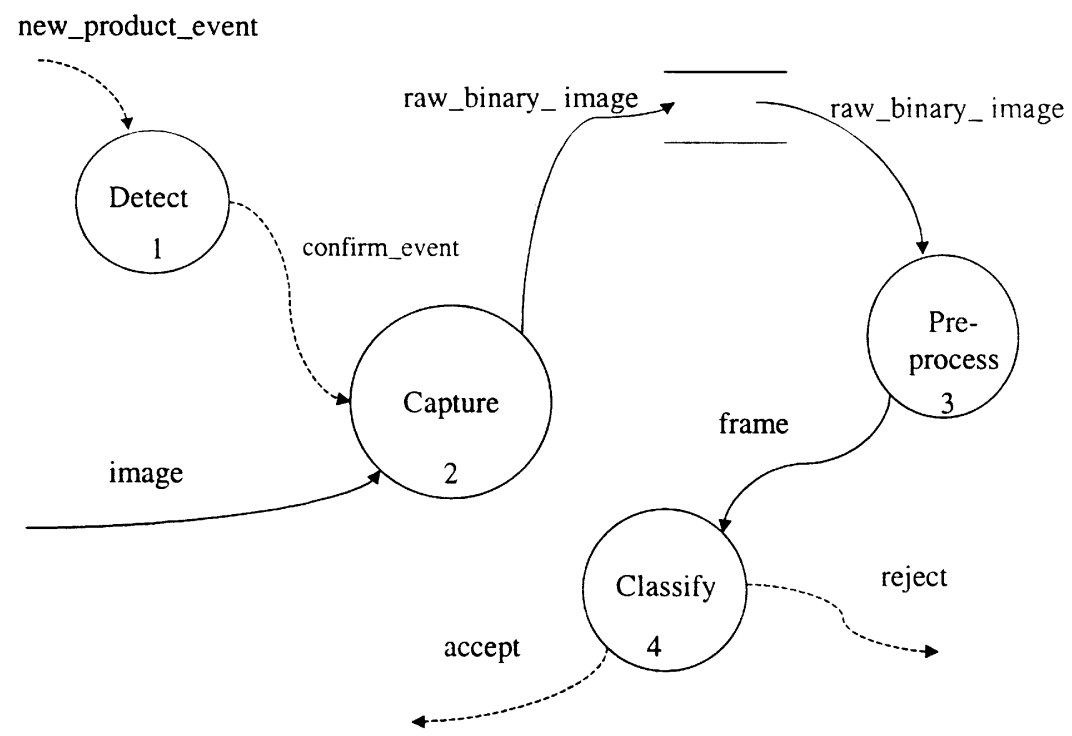

Fig. 4. The level 0 dataflow diagram for the Visual Inspection System.

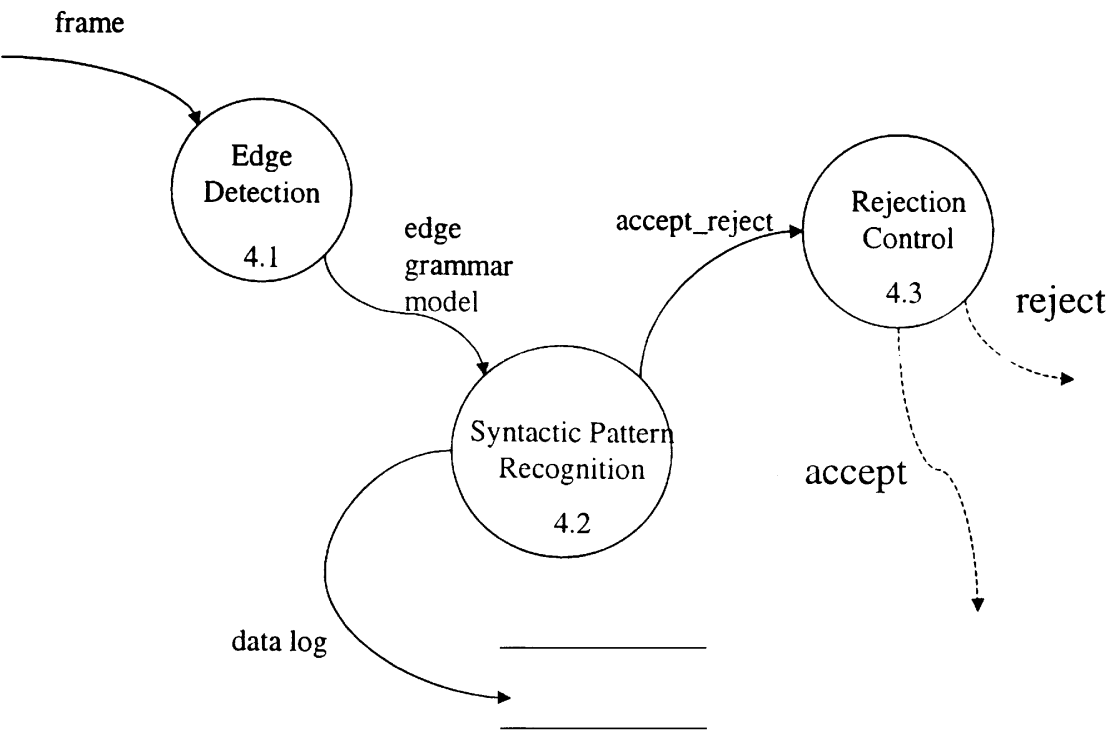

Fig. 5. The level 1 DFD for process 4, classification, of the VIS.

Proceeding to the next level provides more detail for Processes 1-4. Process 1 is essentially an interrupt service routine assigned to a photo-diode detector that senses when a new product for inspection reaches the designated point on the conveyer. Process 2 is a buffering routine whose characteristics depend on the specifications of the camera. Hence, without knowing these details it is not possible to go deeper into the design.

Fig. 5 depicts the level 1 DFD for Process 4. Notice how the internal Processes 4.1, 4.2 and 4.3 are labeled to denote that they are a finer degree of detail of Process 4 shown in the 0 level diagram. Successive levels of detail will follow a similar numbering system (e.g. 4.1.1, 4.1.2).

In addition to the DFDs, SD uses a Data Dictionary to document and control interfaces. ERDs are frequently used to define the relationship between the components of the system-much as in the objectoriented paradigm. The Data Dictionary documents each interface flow in the DFD. Data Structure Diagrams are also used to describe information about logical relationships in complex data structures. The 
Entity Relationship model (which is optional) and Data Dictionary for the Visual Inspection System are not shown for brevity.

\subsection{Real-time extensions of SASD}

It is well known that the standard SASD methodology is not well equipped for dealing with time given its data-, rather than process-oriented perspective. In order to address this shortcoming, Hatley and Pirbhai [15] extended the SASD method by allowing for the addition of control flow analysis. To do this the following artifacts were added to the standard approach:

- directed dashed lines indicate the flow of control information, and

- solid bars indicate "stored" control commands (control stores), which are left unlabeled.

The addition of the new control flows and control stores allow for the creation of a diagram containing only these elements called a Control Flow Diagram (CFD). These CFDs can be decomposed into C-SPECs (control specifications), which can then be described by in STD.

Although the Hatley-Pirbhai extensions recommend that the CFD and C-SPECs stand alone, the CFD by itself makes little sense (as in Fig. 6). Hence, the CFD and DFD are generally combined as shown in Figs. 4 and 5 .

While the Hatley-Pirbhai extensions allow a closer relationship between the DFDs and STDs in a specification, there is still an obvious lack of timing information. Even the simplest temporal characteristic such as response time is unspecified.

\section{Level 0 CFD}

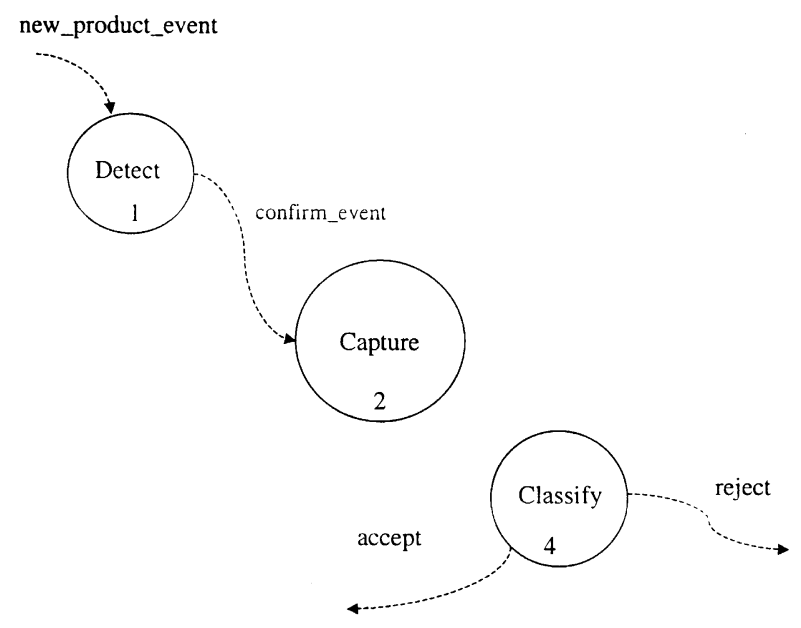

Fig. 6. A CFD for level 0 for the VIS.

\subsection{Problems with SASD in real-time imaging applications}

There are several apparent problems in using SASD to model the Visual Inspection System, not least the difficulty in modeling time and events. For example, what if the inspection system captures a new image in parallel with preprocessing of the last image capture? ${ }^{1}$ Concurrency is not easily depicted in this form of SASD.

Another problem arises in the Context Diagram. Control flows, such as "reject" and "accept" are not easily translated directly into code because they are hardware dependent. In addition, the CFD can sometimes lack clarity since there is no connectivity between portions of it, a condition known as "floating".

Details of the detector and camera hardware also need to be known for further modeling of Process 1 . What happens if the hardware changes? What if a different strategy for classification in Process 2 is needed? In the case of Process 3, (preprocessing) what if the algorithm or even the sensitivity levels change because of the installation of new hardware? In each case the changes would need to propagate into the level 1 DFD for each process, any subsequent levels and, ultimately, into the code.

Clearly making and tracking any of these changes is fraught with danger. Moreover, any change means that significant amounts of code would need to be rewritten. It would seem, then, that this approach does not easily implement the characteristics expressed earlier as recurring issues facing imaging system development; the system lacks robustness in response to minor changes in requirements or operating environment, the design lacks flexibility because generality is difficult to implement systemically and the system is too tightly coupled and dependent upon the underlying platform.

\section{Object-oriented analysis and design (OOAD) using the UML}

The Unified Modeling Language (UML) is widely accepted as the de facto standard language for the specification and design of software-intensive systems [16]. By bringing together the "best-of-breed" in specification techniques the UML has become a family of languages (diagram types) and users can choose which members of the family are suitable for their domain.

The UML is a graphical language based upon the premise that any system can be decomposed into a community of interacting entities and that various

\footnotetext{
${ }^{1}$ This scenario would be desirable if, for example, the reject mechanisms were further down the inspection line and the conveyor systems were running at a high rate.
} 
aspects of those entities, and their communication, can be described using the set of nine diagrams: Use Case, Sequence, Collaboration, Statechart, Activity, Class, Object, Component and Deployment. Of these, five render behavioral views (Use Case, Sequence, Collaboration, Statechart and Activity) while the remaining are concerned with architectural or static aspects.

With respect to real-time systems it is these behavioral models that are of interest. The Use Case Diagrams document the dialog between external actors and the system under development, Sequence and Collaboration Diagrams describe interactions between objects, Activity Diagrams illustrate the flow of control between objects and Statecharts represent the internal dynamics of active objects.

The principal artifacts generated when using the UML and their relationships are shown in Fig. 7.

While not aimed specifically at real-time system design, some notion of time has been included in the UML and at least two of its primary authors $[17,18]$ have proposed approaches for real-time system development. Of the two methodologists, Jacobson has been the most descriptive in terms of the handling of real-time issues - indicative of his background in telecommunication systems. The approach suggested by Jacobson [18] is to document time constraints by associating time attributes to event sequences in the Use Cases of a system. As the development progresses these requirements are transferred into Sequence Diagrams and finally on to the relevant objects. The total time required for each object to complete its tasks is checked to be less than or equal to the time associated to the Use Case sequence. No analysis technique is provided to verify the temporal performance, however. Instead, Shaw's approach [19] is suggested where the minimum and maximum execution times for each task, as well as some allowance for control and actuation delay, referred to as environment overhead, are used to determine predictability.

Douglass [20] builds substantially on Jacobson's approach with his Rapid Object-Oriented Process for Embedded Systems (ROPES). This provides a detailed description of the issues involved in embedded system development as well as considerable discussion on how to pictorially represent the intentions of the developer with the UML notation.

In a separate initiative Selic [21] has modified his Real-Time Object-Oriented Modeling language (ROOM) [22] to align with the UML. This approach, termed "UML for Real-Time", introduces new stereotypes to the UML to support the modeling of complex real-time systems, and describes dynamic behavior with Statecharts.

\subsection{Modeling time explicitly}

It is clear from the description above that the UML in its current form does not provide sufficient facilities for

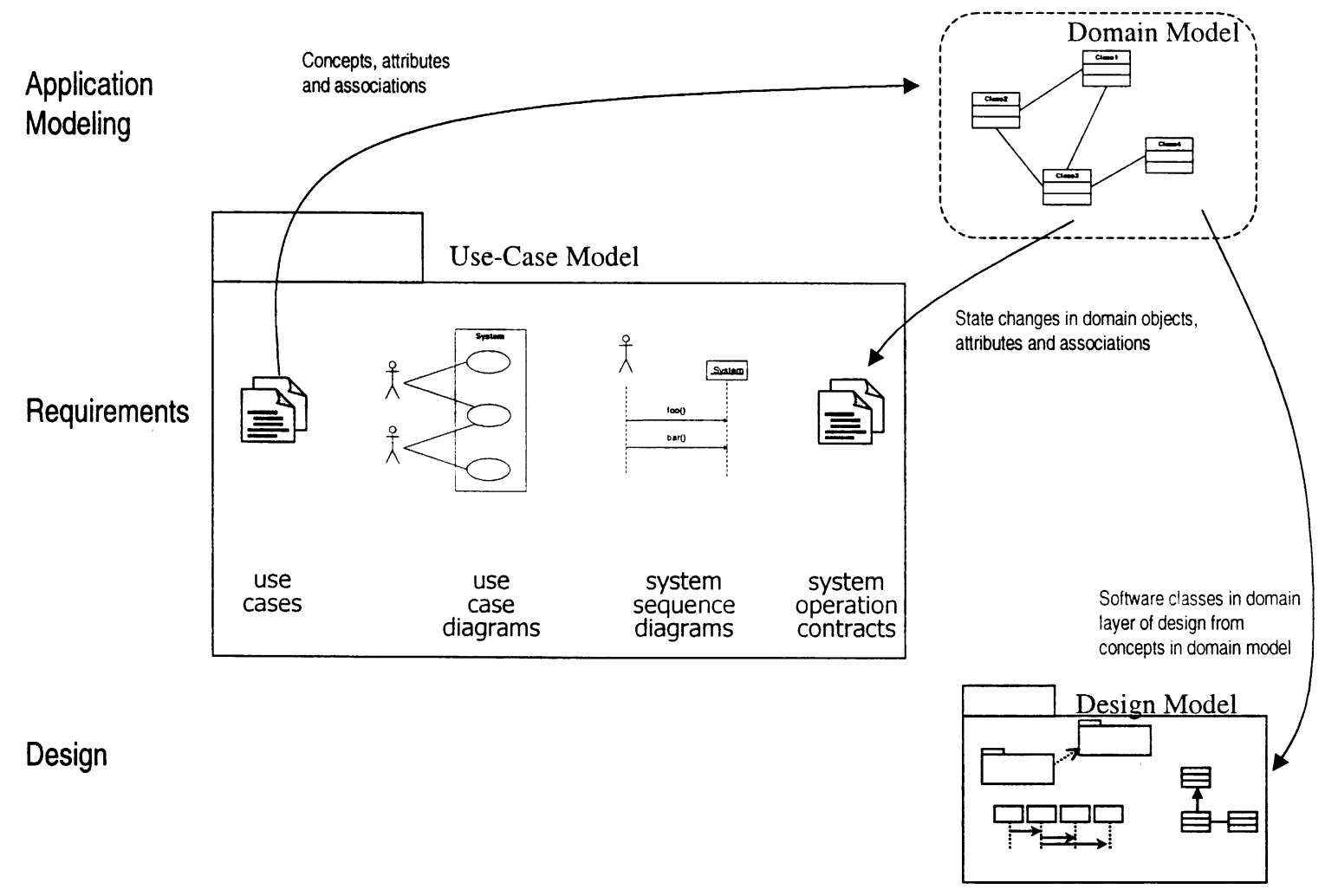

Fig. 7. The UML and its role in specification and design. Adapted from [16]. 
the specification and analysis of real-time systems. It is also stated, however, that the UML is a family of languages and there is no compelling reason for not adding to the family if a suitable language is found. Unfortunately, the majority of appropriate candidates are formal methods - specification languages with a sound mathematical basis - and these are traditionally shunned by the user community. An approach intended to overcome this is described in [23] where the formal model of the system is generated from annotated UML specifications. In this case the formal model chosen is the Q-model.

The Q-model was originally developed by Quirk and Gilbert [24] at the U.K Atomic Energy Research Establishment and was intended as a formal method for the description of temporal characteristics of complex real-time systems. The method was advanced by Motus and a series of prototype tools was developed to support model simulation and verification [25]. The Q-model is based upon the concept that a real-time computer system is composed of a set of loosely coupled, repeatedly activated, terminating processes. The couplings, termed channels, define the synchronization properties of the inter-process communication, as well as the time-selectivity of data transfer between processes. The resultant model can then be checked for completeness, non-contradiction and correctness as well as for timeliness, liveness and constraint conformance. To help to make this crucially important topic more widely known and properly understood, Appendix A, which contains a glossary of terms, is provided for convenient reference. These terms are also described as they are introduced in the foregoing discussion.

\subsubsection{Temporal parameters}

The crucial aspect of the Q-model that makes it suitable for the specification and design of real-time systems is the ability to specify and subsequently verify and validate the temporal characteristics of the proposed system. To achieve these goals a number of process parameters must be defined (illustrated graphically in Fig. 8 where $P_{1}$ and $P_{2}$ represent two processes):

- Process timeset - defines the set of all start times for a process. This set can be expressed both by extension and comprehension, or can be linked to an external event or to the timeset of another process.

- Process start period $\left(\tau_{p}\right)$-Every process that does not have its timeset defined by another process (has no synchronous or sequential channel inputs) must have a start period defined.

- Process execution time $\left(\tau_{x}\right)$-Every process must have an interval defined that describes the best- and worst-case execution times.

- Data consumption time $\left(\tau_{d}\right)$-An interval can be defined, for any process that consumes data that

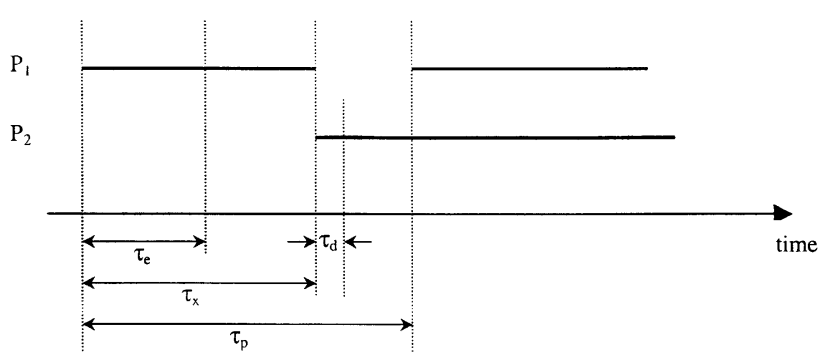

Fig. 8. Temporal parameters of individual processes.

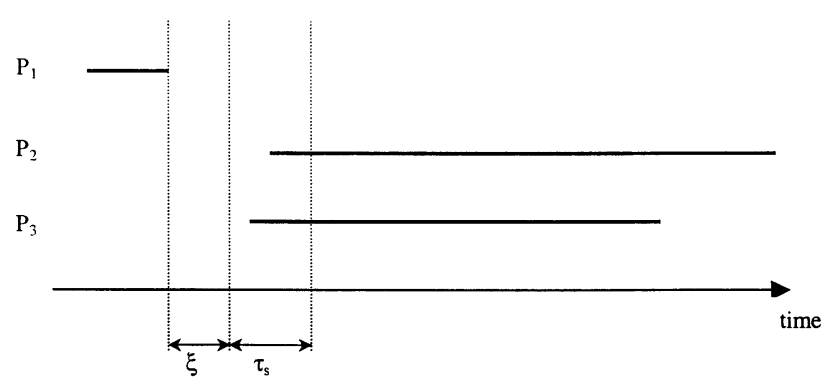

Fig. 9. Temporal parameters of synchronous clusters.

describes an interval that must elapse before data is consumed by that process. Again, this parameter is defined by minimum and maximum values.

- Equivalence interval $\left(\tau_{e}\right)$-This is defined as the time interval during which all occurring events can be considered to have occurred simultaneously. A sideeffect of this is that the equivalence interval defines the time that must elapse before a process can be reactivated. [26].

In addition, there are two further parameters that apply to groups of processes called synchronous clusters illustrated in Fig. 9. A synchronous cluster is formed when two or more processes are linked with synchronous channels such that they all activate at the same time instant. This simultaneous activation is physically impossible, however, so the two parameters are defined to specify the interval during which all of the cluster processes will activate.

- Null channel delay $(\xi)$-This represents the time necessary to detect the synchronizing event and then activate the processes (as this would likely be a broadcast mechanism).

- Simultaneity interval $\left(\tau_{s}\right)$-This is the interval, following the null channel delay, within which all the synchronized processes will be activated.

\subsubsection{Model analysis}

Once the Q-models have been constructed and the parameters specified formal analysis can be performed for verification and simulations can be run for validation. 
There are three levels to the analysis of a Quirk model specification. These are checks on:

1. Individual process specifications - This is the simplest level of analysis and is concerned only with the time parameters for each process. This includes checking that every process has a defined timeset, or a pointer to a timeset via a channel; that the elements of the timeset are well ordered; that a valid channel function has been specified and that the other parameters have been set (execution time, equivalence interval, data consumption time, etc.).

2. Process-pair interaction-All communication within a Q-model is via channels. Several types of channel have been defined and each of these types imposes different rules on the inter-process communication. For each group of synchronously activated processes (synchronous clusters) the null channel delay and simultaneity interval must be determined (from the dynamic properties of the environment). The analysis then verifies that dependencies and channel functions do not violate rules concerning processes waiting for input data or having their process timesets altered. For groups of sequentially activated processes the analysis is more complicated since the consumer process timeset is generated by the producer process and one consumer process could conceivably have many producer processes. In this situation the equivalence interval determines whether the consumer process can be activated (successive activations of the process will be inhibited until the interval has elapsed). For asynchronously connected processes the pairwise analysis is only interested in the estimation of delays that occur during communication.

3. Process group behavior-This is the most complex level of analysis performed on the Q-model. It is here that information deadlocks ("circular" message-waiting conditions) are checked for in sequential chains and synchronous loops, message transfer paths are analyzed and the time required for data to pass through each path is calculated to verify the time constraints imposed upon the designed system by the environment.

This is only a brief overview of the analysis capabilities of the Q-model, however, and is provided here to highlight the usefulness of the approach. A thorough treatment is given in [7].

\subsection{Proposed hybrid methodology}

Given the utility of a formal method such as the Q-model the obvious next question is why use the UML, or any other less formal modeling language? There are many answers to this question ranging from the need for uniform communication standards (and the predominance of the UML in that arena), to the lack of appreciation and willingness of practitioners to adopt rigorous representations (in practical terms very few software development professionals possess the appropriate mathematical skills).

To overcome these issues, yet still maintain rigor, an approach described in [23] is used to transform the behavioral UML models into Q-model structures. This transformation diminishes the exposure of the Q-model to the user and allows for an essentially standard object-oriented development process. Hence, the system specification begins with the representation of the externally accessible functionality as Use Cases.

\subsubsection{Requirements as use cases}

Use Cases are an essential artifact in OOAD and are described graphically in the Use Case Diagram. This can be considered analogous to the Context Diagram in SASD in that it represents the interactions of the software system with its external environment.

Use Cases are represented graphically as ellipses, as can be seen in Fig. 10. Each Use Case is, however, a document that describes scenarios of operation of the system under consideration as well as pre- and postconditions, and exceptions. It is proposed that in the specification of a real-time system this is also where overall time constraints, sampling rates and deadlines are specified.

In an iterative development lifecycle the Use Cases will become increasingly refined and detailed as the analysis and design workflows progress. Interaction diagrams are then created to describe the behaviors defined by each use case. In the first iteration these diagrams depict the system as a "black box", but once Domain Modeling has been completed the black box is transformed into a collaboration of objects as will be seen later.

As stated above, the Domain Model is created based upon the Use Cases and, through further exploration of system behavior via the Interaction Diagrams, the Domain Model evolves systematically into the Design Class Diagram. The construction of the Domain Model is, therefore, analogous to the analysis stage in SASD described earlier. In Domain Modeling the central objective is to represent the real-world entities involved in the domain as concepts in the Domain Model. This is a key aspect of object-oriented systems and is seen as a significant advantage of the paradigm since the resultant model is "closer" to reality than in alternative modeling approaches, including SASD. Part of the design class diagram that results from evolution of the Domain Model is shown in Fig. 11. 


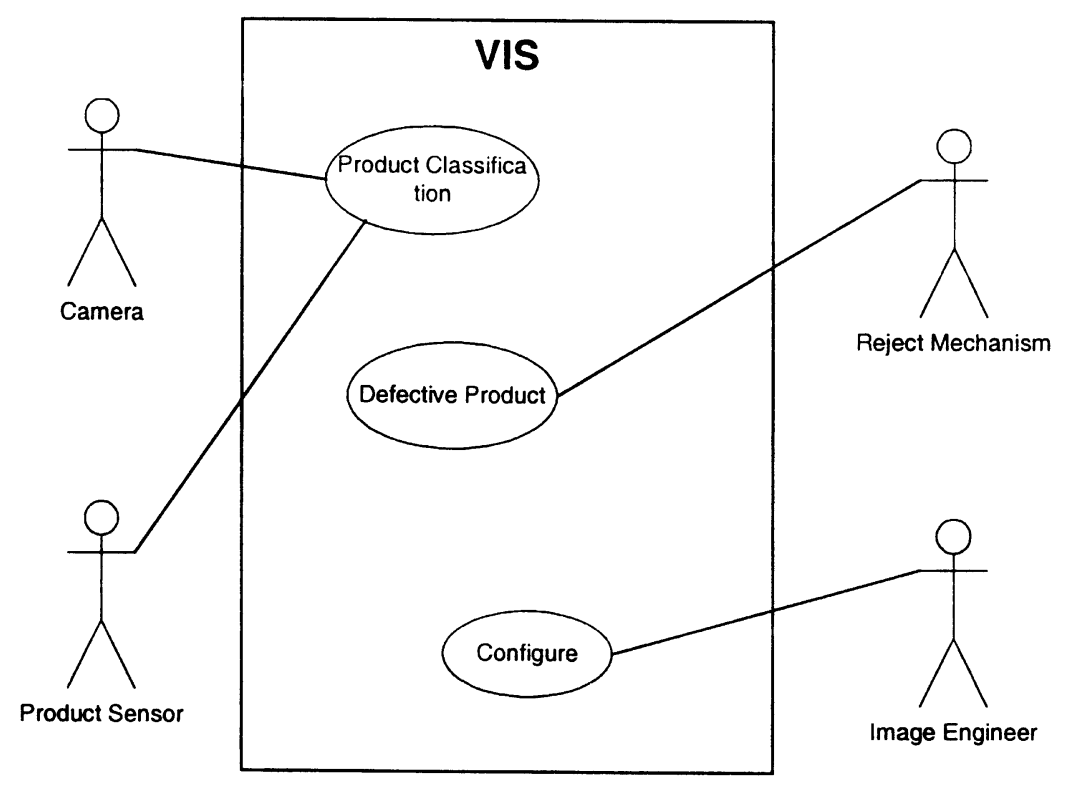

Fig. 10. Use Case Diagram of VIS.

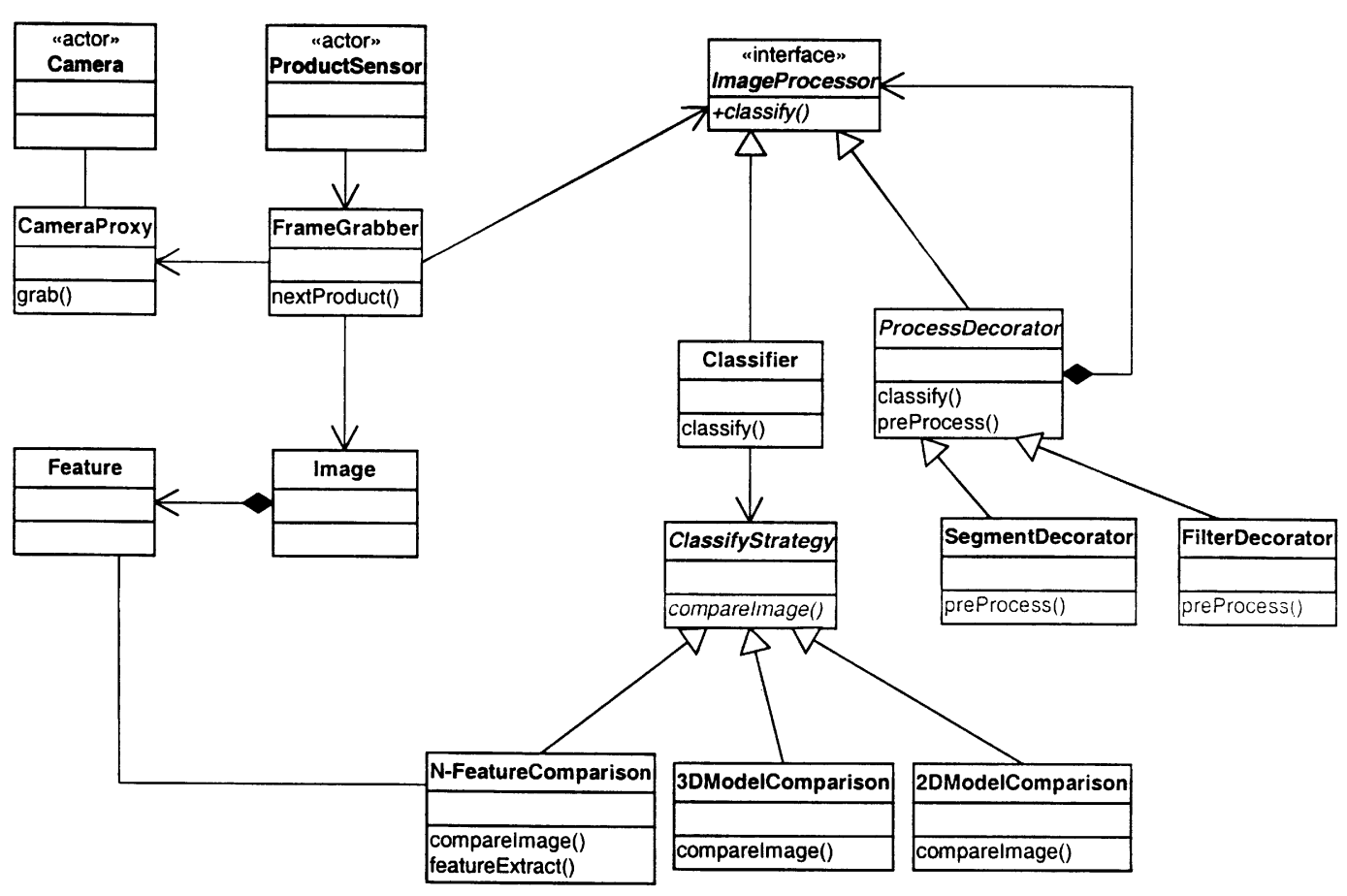

Fig. 11. Partial Design Class Diagram of AVI System.

\subsubsection{Structural design}

The design class diagram is used to show the static structural view of the system by describing the classes of objects that will comprise the software solution. As can be seen in Fig. 11 the system is composed of image capture elements (CameraProxy, FrameGrabber) and image classification elements (Classifier, ImageProcessor, "Decorators" and "Strategies"). The decorators and strategies are aspects of the design introduced by applying well-known design patterns from [6].

The principal aim of these patterns is to allow for dynamic (i.e. runtime) changes to the pre-processing and classification schemes. This is achieved by abstracting invariant behavior into supertypes (ImageProcessor, ProcessDecorator and ClassifierStrategy) and allowing subtypes to implement variant behavior (N-FeatureComparison, FilterDecorator). It can be 
argued that this generality is speculative, and therefore overcomplicates the design, but the intent of this work is to build a framework for real-time image processing systems where this generality is critical for reuse and, indeed, characterizes the advantage of using OOAD. Through careful abstraction and inheritance a core framework of services can be created that is easily extended for different applications, platforms, algorithms and devices. This variation by extension rather than modification (to the core services) protects the overall structure and architecture of the system, making it robust, yet allows for changing requirements and advancements, making it flexible and reusable thus fulfilling all of the desires expressed earlier.

\subsubsection{Behavioral modeling}

Returning to the case study, the sequence diagram shown in Fig. 12 represents the ordering of messages between objects in the system in response to the arrival of the next product on the conveyor. It is clear that the nextProduct message is generated from the external sensor that triggers the FrameGrabber. The Image created by the output from the Camera (via the CameraProxy) is pre-processed using one of the decorators (FilterDecorator) and then classified using a classification strategy ( $\mathrm{N}-\mathrm{FeatureComparison).} \mathrm{The}$ result of the classification is then sent to the RejectController that logs the result and triggers the rejection mechanism if required.

It is evident from these diagrams that no timing information is included beyond the logical timing (ordering) of the messages in the sequence diagram. This is insufficient for real-time systems as stated earlier. Not only are we unable to prove the correctness of the temporal specification, but we have, indeed, neglected to represent a critical aspect of the domain in our model. It is in this regard that the approach proposed here offers significant reward. To remedy the situation we generate the additional formal model; the Q-model. This is generated from the UML models of the system (with additional information regarding timing properties and constraints).

\subsubsection{Q-model for the AVI}

The Q-model generated from the sequence diagram in Fig. 12 is shown in Fig. 13. The transformation rules applied here are described in [23] but basically constitute a mapping of activations to Q-model processes and messages to Q-model channels. The description of the processes and channels in this Q-model are presented in Tables 1 and 2, respectively, and are representative of typical timing information for AVI systems.

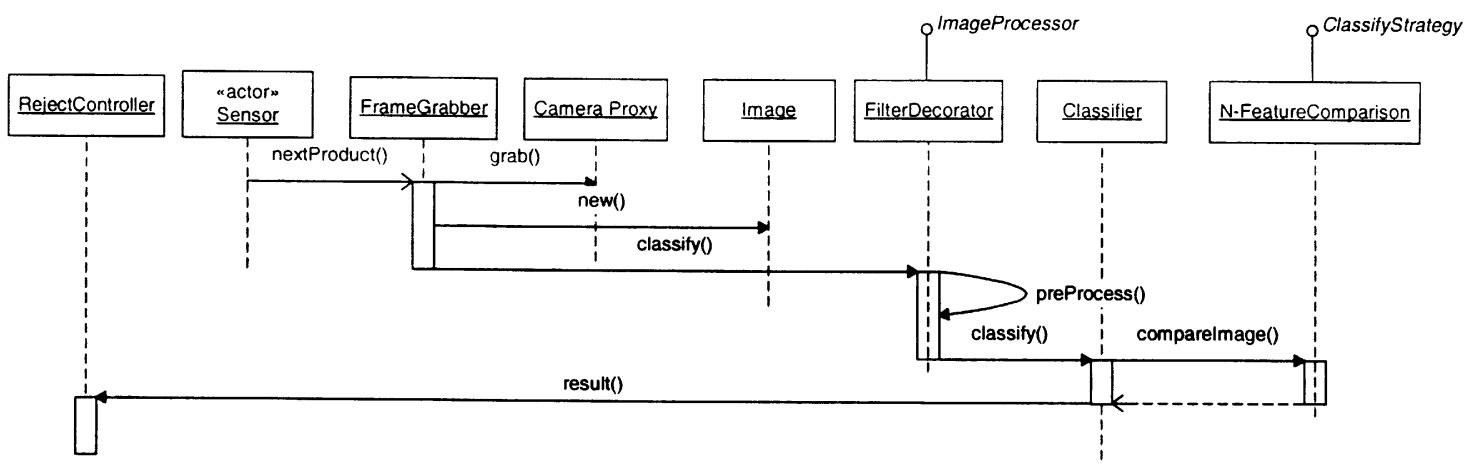

Fig. 12. Sequence Diagram of Product Classification.

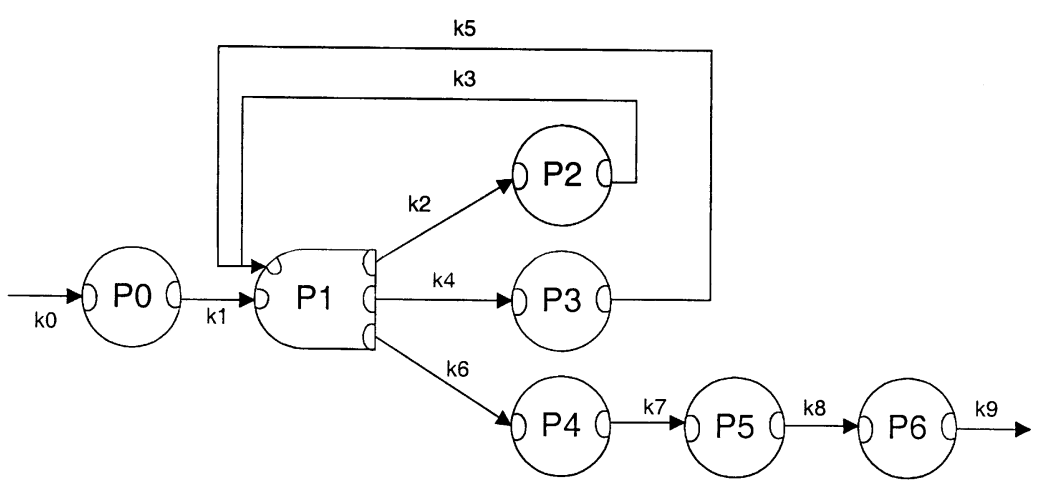

Fig. 13. Q-model of Product Classification. 
Table 1

Description of Product Classification Q-model

\begin{tabular}{llllrr}
\hline Process & Name & $\tau_{p}(\mathrm{~ms})$ & $\tau_{x}$ & \multicolumn{1}{c}{$\tau_{d}$} & $\tau_{e}$ \\
\hline P0 & Null process (sense event) & 33 & 0 & 0 & 31 \\
P1 & nextProduct( ) & - & $1-2$ & 0 & 1 \\
P2 & grab() & - & $5-7$ & & 1 \\
P3 & new() & $4-5$ & $3-4$ & 4 \\
P4 & classify().preProcess ( ) & - & $6-8$ & $3-4$ & 4 \\
P5 & -classify() & - & $1-2$ & 0 & 1 \\
P6 & compareImage( ) & - & $7-10$ & $3-4$ & 4 \\
\hline
\end{tabular}

Table 2

Channel descriptions for Product Classification Q-model

\begin{tabular}{lll}
\hline Channel & Type & Function \\
\hline K0 & Sequential & {$[0,0]$} \\
K1 & Sequential & {$[0,0]$} \\
K2 & Sequential null & - \\
K3 & Sequential & {$[0,0]$} \\
K4 & Sequential & {$[0,0]$} \\
K5 & Sequential & {$[0,0]$} \\
K6 & Sequential & {$[0,0]$} \\
K7 & Sequential & {$[0,0]$} \\
K8 & Sequential & {$[0,0]$} \\
K9 & Sequential & {$[0,0]$} \\
\hline
\end{tabular}

Initially all channels are selected as sequential (the source process triggers the target process upon completion) except for $\mathrm{K} 2$ which is sequential null indicating that no data is transferred. The alternatives are synchronous (where the timesets of the producer and consumer processes are identical) and asynchronous (the producer and consumer processes have independent timesets - data is transferred but there is no triggering of activation). The channel functions indicate the range of data generations that are consumed by the process (allowing for time-selective communication if necessary) but here all are set to the most recent generation only. During simulation the channel types and functions can be altered to examine the effect of parallelizing aspects of the design.

Each Q-model process can then be decomposed into a separate Q-model such as the one shown in Fig. 14 for process $\mathrm{P} 6$, the compareImage process. In this case elements of the Q-model structure are transformed from the ClassifyStrategy hierarchy but individual algorithmic differences must be constructed directly. The fragment shown in Fig. 14 is the Q-model of the $\mathrm{N}-$ FeatureComparison object. The bubbles represent processing tasks and the directed arcs represent the channels that connect and coordinate the tasks.

Once the Q-model representation is complete the model can be analyzed as described earlier. Typical time line diagrams that are generated during simulation are shown in Figs. 15 and 16. It is clear from Fig. 15 that if

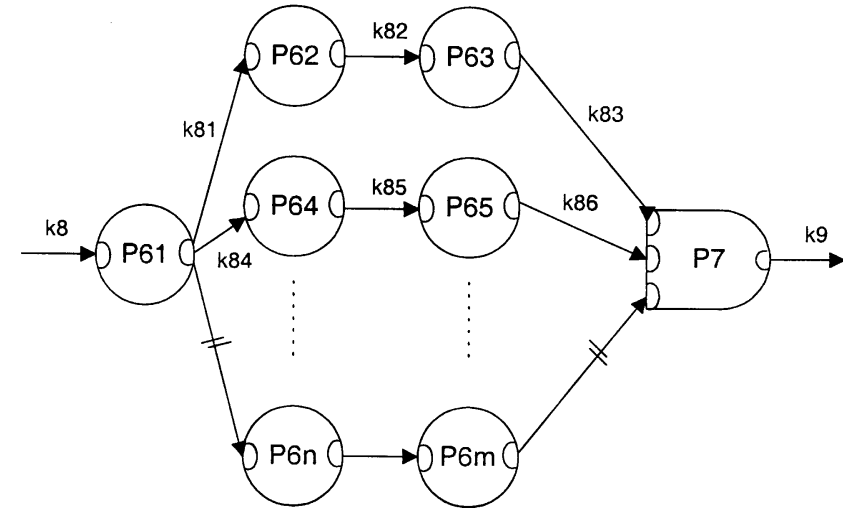

Fig. 14. Q-model of compareImage for N-FeatureComparison classification "strategy".

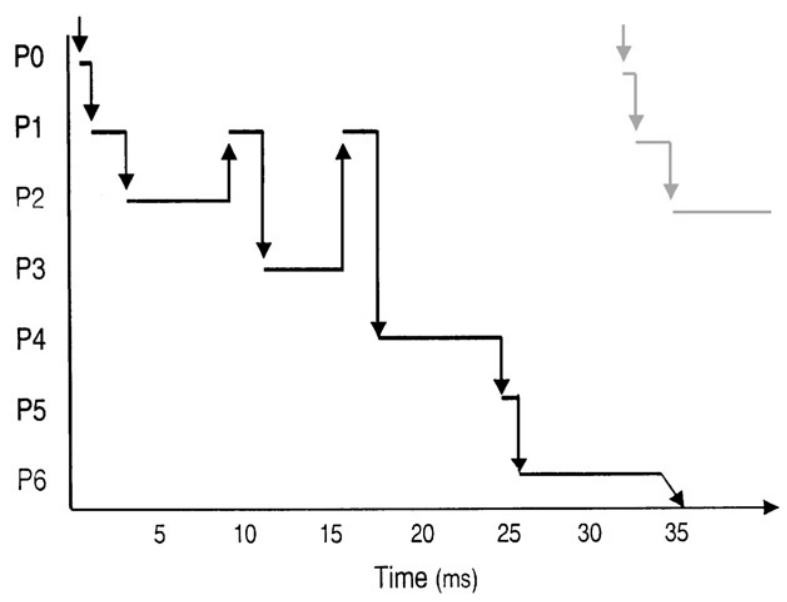

Fig. 15. Simulation timeline for Product Classification Q-model.

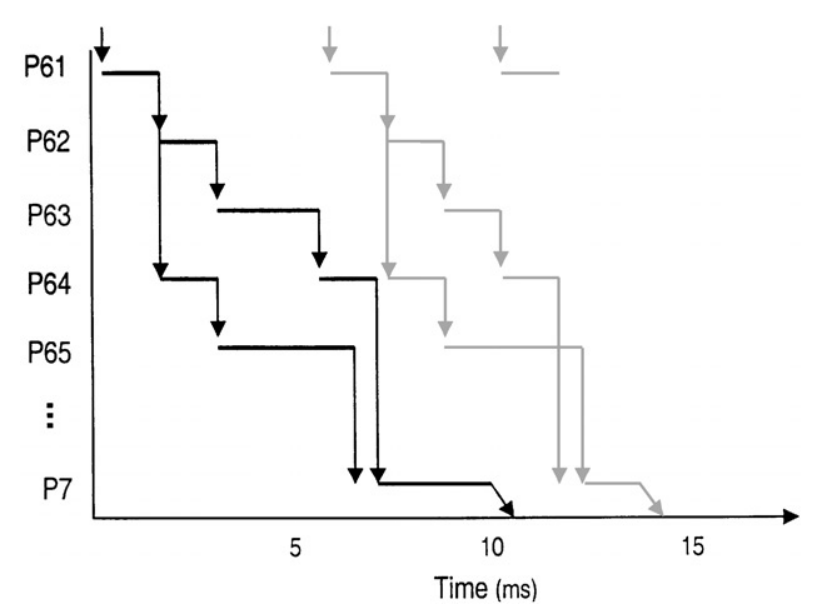

Fig. 16. Simulation timeline for $\mathrm{N}-$ FeatureComparison process $(n=2)$.

the process chain P4-P6 was on a separate processor the arrival rate could increase to every $20 \mathrm{~ms}$.

In the case of Fig. 16, the compareImage process is triggered by the arrival of a pre-processed image which 
is then subject to two concurrent feature extraction and feature matching chains, the results of which are aggregated in process P7. Since the temporal properties of each process are defined the simulation can highlight the minimum and maximum arrival rate based upon the available resources.

\section{Conclusions}

In this paper, we have presented an approach to the specification of real-time imaging systems using a prototypical visual inspection system as a case study. Such systems are typically developed by imaging engineers who have traditionally been reticent of using more modern approaches to software development; often favoring legacy, or their own individual, techniques. This approach does not lend itself well to robust, maintainable, extensible and reusable system development, however, so an alternative is sought.

The alternative presented here is based upon the UML since this is widely regarded as the industry standard for the specification and design of software intensive systems. It is clear, however, that in many realtime system applications, including many imaging systems, any specification and design models must be analyzed formally to ensure that the temporal performance imposed by the environment is obtainable in the intended scheme. To this end a formal method, the Q-model, is introduced. The formal models can be generated from the UML specifications so should not deter potential users, yet still provide the rigor appropriate to the domain.

An example was presented to illustrate the proposed approach based upon an industrial visual inspection system. At a superficial level perhaps little advantage can be seen for using the hybrid objectoriented approach described here over conventional structured techniques, but through the application of some subtle design principles it is apparent that the object-oriented specification can better accommodate future changes that are typical in all software systems. In addition the OO design principles have been codified into a set of reproducible design patterns that make the application of those principles simpler and more efficient.

The authors are working on the design and eventually construction of a framework for image processing systems using the UML-based approach described here. This will allow efficient reuse of standard image processing services such as compression, image preprocessing and multimedia manipulation through specific extension points within the framework and will provide confidence for real-time applications through the use of the Q-model.
Appendix A. Glossary of terms relating to the Q-model

Asynchronous channel - the producer and consumer processes timesets are independent. There is no synchronization between the processes.

Channel - a point-to-point one-way communication between repeatedly activated, terminating producer and consumer process that defines the synchronization properties between them.

Channel function - the "history" of data generations of a producer process that is available to the consumer process. A channel can be thought of as a logical buffer and the channel function would be the buffer's capacity. This allows for time-selective communication, the notion that a consumer process might not require the most recent generation of data, but rather an earlier generation.

Coupling - see channel.

Null channel - provides synchronization between processes when data is not transferred.

Null channel delay-time interval to detect the synchronizing event and then activate the associated processes.

Process - an atomic unit of computation, whose terminal behavior is specified, but whose internal operation is unspecified. Depending on the application, a process can be an instruction, a procedure or even an entire system.

Process execution time - an interval that describes the best- and worst-case execution time for a process.

Simultaneity interval - the time interval, following the null channel delay, within which all the synchronized processes will be activated.

Synchronous cluster - two or more processes that are linked by synchronous channels such that they all activate at the same time instant.

Process timeset - the set of all activation instants of a process. An explicit description of process start times.

Process start period - activation instant for a process whose timeset is not defined by another process.

Synchronous channel-the producer and consumer processes timesets are identical. Practically this means that producer and consumer processes are activated simultaneously.

Sequential channel - the consumer process timeset is generated from the producer process timeset. Practically this means that the consumer process is activated when the producer process terminates.

\section{References}

[1] Laplante PA. A retrospective on real-time imaging, a new taxonomy and a roadmap for the future. Real-Time Imaging 2002;8(5):413-25. 
[2] Laplante PA, Neill CJ. An overview of software specification techniques for real-time imaging. In: Proceedings of the RealTime Imaging Conference, SPIE, San Jose, January 2002. p. 55-64.

[3] Dougherty ER, Laplante PA. Real-time image processing. Bellingham, WA: SPIE Press/IEEE Press, 1995.

[4] Laplante PA, Stoyenko A. editors. Real-time image processing: theory, techniques, and applications. Piscataway, NJ: IEEE Press, 1996.

[5] Davies ER, Ip HHS. Guest editorial: real-time visual monitoring and inspection. Real-Time Imaging 1998;4(5):313-5.

[6] Gamma E, Helm R, Johnson R, Vlissides J. Design patterns: elements of reusable object-oriented software. Reading, MA: Addison-Wesley, 1994.

[7] Motus L, Rodd MG. Timing analysis of real-time software. Oxford: Pergamon, 1994.

[8] Thomas ADH, Rodd MG, Holt JD, Neill CJ. Real-time industrial inspection: a review. Real-Time Imaging 1995;1(2):139-58.

[9] Parnas DL. On the criteria to be used in decomposing systems into modules. Communications of the ACM 1972;15(12):1053-8.

[10] Parnas DL. Introduction to chapter 6.5. In: Laplante PA, editor. Great papers in computer science. New York: West Publishing, 1996.

[11] Yourdon E, Constantine L. Structured design. Englewood Cliffs, NJ: Prentice-Hall, 1975.

[12] DeMarco T. Structured analysis and system specification. Englewood Cliffs, NJ: Prentice-Hall, 1978

[13] Ward PT, Mellor SJ. Structured development for real-time systems, vol. 1: introduction \& tools; vol. 2: essential modeling techniques; vol. 3: implementation modeling techniques. New York, NY: Yourdon Press, 1985.
[14] Yourdon E. Modern structured analysis. Englewood Cliffs, NJ: Prentice-Hall, 1991

[15] Hatley DJ, Pirbhai IA. Strategies for real-time system specification. New York, NY: Dorset House Publishing Co., 1987.

[16] Larman C. Applying UML and patterns: an introduction to object-oriented analysis and design and the unified process, 2nd ed.. Englewood Cliffs, NJ: Prentice-Hall, 2002.

[17] Booch G. Object-oriented design with applications. Menlo Park, CA: Benjamin/Cummings, 1991.

[18] Jacobson I, Christerson M, Jonsson P, Overgaard G. Objectoriented software engineering: a use case driven approach Reading, MA: Addison-Wesley, 1992.

[19] Shaw AC. Reasoning about time in higher-level language software. IEEE Transactions on Software Engineering 1989;15(7):875-89.

[20] Douglass BP. Real time UML - developing efficient objects for embedded systems. Reading, MA: Addison Wesley, 1998.

[21] Selic B, Rumbaugh J. Using UML for modeling complex realtime systems. ObjecTime Limited/ Rational Software Corp. white paper, March 1998. Available at www.rational.com

[22] Selic B, Gullekson G, Ward PT. Real-time object-oriented modeling. New York: Wiley, 1994.

[23] Neill CJ, Holt JD. Adding temporal modeling to the UML to support systems design. Systems Engineering 2002;5(3):213-22.

[24] Quirk WJ, Gilbert R. The formal specification of the requirements of complex real-time systems. Atomic Energy Research Establishment, Harwell, UK, No. 8602, 1977.

[25] Naks T, Motus L. Handling timing in a time-critical reasoning system - a case study. Annual Reviews in Control 2001;25:157-68.

[26] Palouja R. Embedded software specification and analysis. Ph.D thesis, University of Wales, 1992. 